\title{
Si Multidot FETs for Single-Electron Transfer and Single-Photon Detection
}

\author{
M. Tabe* , R. Nuryadi, D. Moraru, Z.A. Burhanudin, \\ K. YOKOI AND H. IKEDA \\ Research Institute of Electronics, Shizuoka University \\ 3-5-1 Johoku, Hamamatsu 432-8011, Japan
}

\begin{abstract}
Recently, there have been increasing demands for controlling individual electrons, photons, and dopants in developing nm scale Si devices. Our most recent results on $\mathrm{Si}$ single-electron nano-devices will be presented. We have demonstrated single-electron transfer in random-tunnel-junctions by a cycle of ac gate bias, detection of photons and detection of individual acceptor ions by Si single-hole transistor.
\end{abstract}

PACS numbers: 73.21.La, 73.23.Hk

\section{Introduction}

Si single-electron-tunneling (SET) or single-hole-tunneling (SHT) devices have a great potential in realizing a variety of new functions. So far, we have fabricated multi-junction Si SET/SHT field-effect transistors (FETs) by a couple of unique but simple methods, in which the channel region has a structure of mutually-connected Si dots or dopant-induced potential. By using these devices, most recently, we have obtained several important results of single-electron transfer, single-photon detection and single-ion (dopant) detection.

In this paper, we will review our recent results, which, we believe, open up new and wide possibilities for future electronics.

\section{Single-electron-turnstile in dopant-induced random junctions}

More than fifteen years ago, single-electron turnstile [1] and single-electron pump [2] devices, consisting of precisely designed multiple-capacitors, were proposed as those that can achieve time-controlled tunneling of individual electrons by means of ac gate voltages. Each cycle of the ac gate conveys exactly one electron from the source to the drain, leading to the resultant current of the circuit,

*corresponding author; e-mail: romtabe@rie.shizuoka.ac.jp 


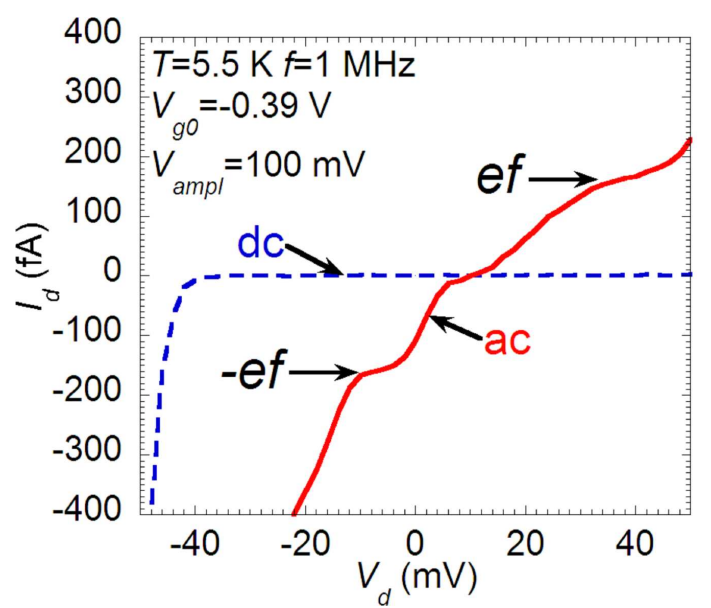

Fig. 1. $I_{\mathrm{d}}-V_{\mathrm{d}}$ characteristics measured at $T=5.5 \mathrm{~K}$ under dc operation (dashed curves) and ac operation (solid curves). Current plateaus appear around \pm ef $( \pm 0.16 \mathrm{pA})$ levels.

$I=e f$. Recently, we have discovered that even random multiple-tunnel capacitors have a capability to transfer electrons one by one $[3,4]$.

Recently, we have found by collaboration with Ono (NTT) that a P-doped Si-nanowire transistor can transfer electrons one by one by means of ac gate bias [4]. This is quite surprising because multiple- tunnel-capacitors (or -junctions) are naturally formed by randomly distributed P-ions and it was not evidenced from the conventional theory that such random junctions have a capability of single-electron turnstile operation. We have analyzed these phenomena by theoretical simulations and found that most of non-homogeneous capacitance arrays statistically lead to the successful turnstile operation with unexpectedly high probabilities. Figure 1 shows measured dc and ac characteristics of SET current vs gate voltages. Plateaus at $I=e f$ are indicating that each ac cycle of gate bias conveyed one electron.

\section{Random-telegraph-signal induced by photon absorption}

Interaction with photons for SETs is another important subject for future applications such as quantum computing, medical imaging and other novel optoelectronic devices. Under light illumination, absorbed photons can change the number of electrons in the dots, sensitively modulating the current level [5]. Figure 2 shows our two-dimensional (2D) multiple-dots (multiple-tunnel junctions) FET, working as an SET device [6]. We have demonstrated $[7,8]$ that this device can detect a single-photon through random-telegraph-signal (RTS) in the SET characteristics, i.e., each current level switching in the RTS is ascribed to a photogenerated charge effect. The RTS, which was wavelength and intensity dependent, occurs when the dot potential changes due to single-photon absorption. 


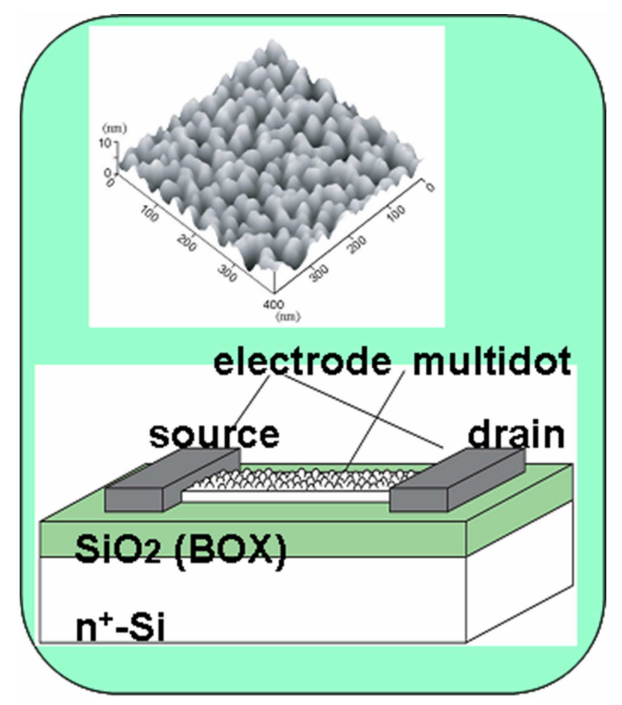

Fig. 2. A schematic view of our multiple-dots FET. The channel part consists of randomly distributed Si dots, as indicated by the atomic force microscopy (AFM) image (upper part).

\section{Single-dopant detection}

In order to improve the quantum efficiency, we have tried to detect photons absorbed in the underlying Si substrate by changing the substrate structure from

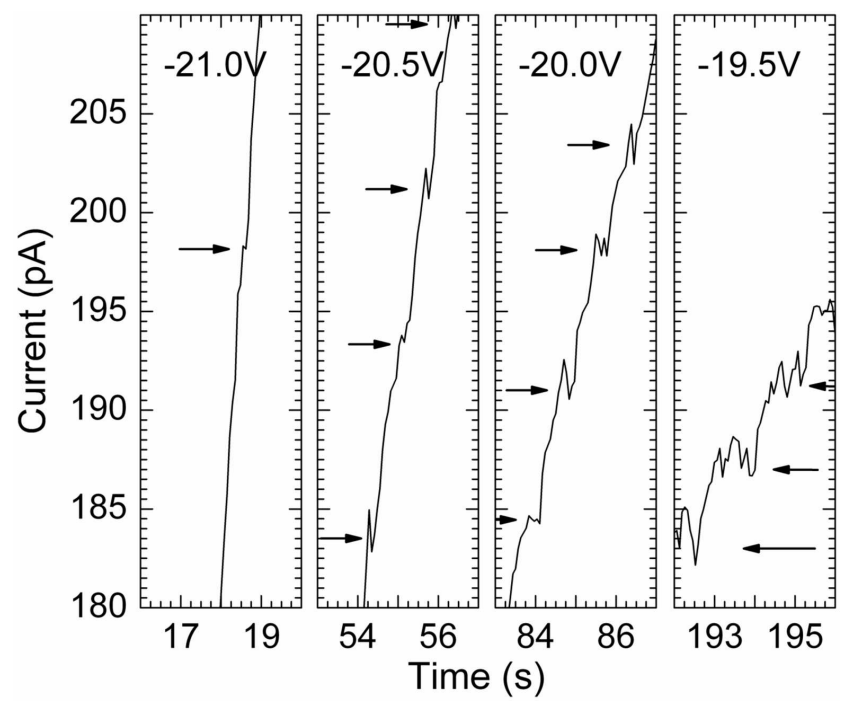

Fig. 3. The typical step-like features (indicated by the arrows) in magnified $I_{\mathrm{d}}-t$ plots. Each step is interpreted as ionization of individual B atoms in the substrate. 
$n^{+}$-Si to $p$-epilayer on $p^{+}$-Si substrate. As a result in the dark condition, we observed step-like features in the drain current $\left(I_{\mathrm{d}}\right)$ vs time $(t)$ plot, as shown in Fig. $3[9]$.

Since the measured temperature was $17 \mathrm{~K}$, most of B atoms are frozen out. By sudden application of a relatively high electric field, B atoms are expected to be ionized sequentially. Thus the observed step-like features are ascribed to individual B ionization [9]. This result encourages us because single-ion resolution guarantees single-photon resolution for absorption in the substrate. Experiments of photoillumination are under way.

\section{Conclusion}

We have demonstrated characteristics of nanometer-scale Si SET devices with respect to interactions with individual dopants and photons. These results will be leading us to new aspects of future devices.

\section{Acknowledgments}

We would like to thank Dr. Y. Ono (NTT) and Dr. H. Inokawa (Shizuoka Univ.) for tight collaboration in research of single-electron transfer, and Mr. T. Mizuno (Shizuoka Univ.) for his continuous support in experiments. This work was partially supported by MEXT KAKENHI (16106006 and 16206038).

\section{References}

[1] L.J. Geerligs, V.F. Anderegg, P.A.M. Holweg, J.E. Mooij, H. Pothier, D. Esteve, C. Urbina, M.H. Devoret, Phys. Rev. Lett. 64, 2691 (1990).

[2] H. Pothier, P. Lafarge, C. Urbina, D. Esteve, M.H. Devoret, Europhys. Lett. 17, 249 (1992).

[3] H. Ikeda, M. Tabe, J. Appl. Phys. 99, 073705 (2006).

[4] D. Moraru, Y. Ono, H. Inokawa, M. Tabe, Phys. Rev. B 76, 075332 (2007).

[5] A. Fujiwara, Y. Takahashi, K. Murase, Phys. Rev. Lett. 78, 1532 (1997).

[6] R. Nuryadi, H. Ikeda, Y. Ishikawa, M. Tabe, IEEE Trans. Nanotechnol. 2, 231 (2003).

[7] R. Nuryadi, H. Ikeda, Y. Ishikawa, M. Tabe, Appl. Phys. Lett. 86, 133106 (2005).

[8] R. Nuryadi, Y. Ishikawa, M. Tabe, Phys. Rev. B 73, 045310 (2005).

[9] Z.A. Burhanudin, R. Nuryadi, M. Tabe, Appl. Phys. Lett. 91, 042103 (2007). 